\title{
Improving Research Training Using Assignments for Family Medicine Residents in Saudi Arabia
}

\section{Mostafa Kofi ${ }^{1 *}$, Saad Albattal ${ }^{1}$, Ghada Alarfaj ${ }^{1}$, Abood Al Abood ${ }^{1}$, Abdelaziz Mansour Bin Alrasheed ${ }^{1}$, Tarek Elsaid ${ }^{1}$, Maha Moharram ${ }^{1}$, Lamiaa Fiala ${ }^{2}$ and Ayman Afifi ${ }^{1}$}

${ }^{1}$ Family and Community Medicine Department, Prince Sultan Military Medical City,

Riyadh, Saudi Arabia

${ }^{2}$ Department of Health Sciences, Faculty of Health and Rehabilitation Sciences, Prin-

cess Nourah Bint Abdulrahman University, Riyadh, Saudi Arabia

*Corresponding Author: Mostafa Kofi, Professor, Family and Community Medicine

Department, Prince Sultan Military Medical City, Riyadh, Saudi Arabia.
Received: November 01, 2021

Published: December 09, 2021

(C) All rights are reserved by Mostafa Kofi., et

al.

\begin{abstract}
Setting: The family medicine training program at PSMMC in Riyadh is a major program with many years of experience in graduating residents of family medicine residency programs. The program is approved by the SCFHS. An average of 20-30 residents graduates each year from SCFHS's training program. Residents in the Saudi family medicine program must complete research training as part of their residency training. However, Residents' feedback emphasized the need for a change in the way research methods are taught. For this reason, assignment-based research training was designed to replace lecture-based training and to improve the research training experience.

Methods: A Qualitative study in which a focus group of eight residents was included before and after implementation of research training change to assignment based.

Results: Before implementation, there was an agreement that research training was considered a burden on residents and not a priority. Careless attitude was common among residents towards performance in the research requirement. There is an improved in commitment and seince of ownership for research after implementation of assignment-based research activities

Conclusion: The use of assignment-based training in research training was effective in changing the perception of the residents towards research training as a requirement from the SCFHS.
\end{abstract}

Keywords: Research Training; Residents; Family Medicine

\section{Introduction}

Assessments and assignments play a crucial role in learning. The completion of an assignment is an opportunity to demonstrate the achievement of intended learning outcomes. Through feedback on assignments, the Family Medicine Residents can measure their achievement of the standards set by the course and the Saudi Commission for Health Specialties (SCFHS).
The Academic Affairs and Training Davison at the Family and Community Medicine Department, at the Prince Sultan Medical City (PSMMC) in Riyadh, Saudi Arabia, ensures that all Residents have an equal opportunity to demonstrate their abilities and to receive appropriate and helpful feedback on their work. Thus, the assignments and assessment style method were designed so instructors can use it in their research methods course to point out 
what is required and expected of both family medicine residents and research instructors.

It is intended in this study to examine the extent to which assignments can help and boost the learning process of family medicine (FM) residents in a course that includes training on research methods during their residency programs.

The research objectives included:

- $\quad$ To document the assignment-based training during the research methods course for FM residents.

- $\quad$ To explain the positive aspects of using assignment-based learning for the development of research methods knowledge and skills among FM residents

An American educator, Malcolm Knowles, defined andragogy in the 1970s as 'the art and science of helping adults learn [1]. He identified six principles of adult learning:

- $\quad$ Adult learners are internally motivated and self-directed. They make choices that are relevant to their learning objectives. They also direct their learning goals with guidance from their mentors. Students need to be empowered to make choices on their own.

- $\quad$ Adults bring life experiences and knowledge to learning experiences. Educators encourage learners to connect past experiences with current knowledge and activities. Educators must know how to relate the sum of the learner's experience to the current learning experiences.

- Adults are goal-oriented: Adult learners aim to acquire relevant and adequate knowledge and for this reason, the intended learning outcomes should be identified. Once the learning goals have been identified, educators must align the learning activities such that these objectives are fulfilled within a certain period.

- Adults are relevancy oriented: Adult learners benefit by relating the assigned tasks to their own learning goals. If the activities they are engaged in, directly contribute to achieving their learning objectives, then they will be inspired and motivated to engage in projects and complete them.

- $\quad$ Adults are practical: Educators need to identify appropriate ways and convert theoretical learning to practical ac- tivities. Work placement is a way for students to apply the theoretical concepts learned inside the classroom to real-life situations. Learning is assisted when appropriate ways of implementing theoretical knowledge in real-life situations are made clear.

- Adult learners like to be respected: Adult learners thrive in collaborative relationships with their educators. Learners become more productive when they're considered by their instructors as colleagues. When their contributions are acknowledged, then they are willing to put out their best work.

Consequently, Assignments and assessment are important aspects of learning. Completing an assignment is an opportunity to demonstrate your achievement. Feedback on assignments provides you with measurement of your achievement in relation to the standards set by the course and the college [2].

Adult Teaching should be different from teaching for Children in many ways; in adults, learning mostly is driven internally (via internal focus of control), self-directed and teaching should build upon their prior knowledge, target their practical experience, focus on solving problems and it will be excellent if it succeeds to engage them $[3,4]$.

Active Learning is the grand meta-principle in adult learning as per Patricia Cross [5]. Many teaching strategies have been invented to foster active learning like the blended learning, small group activities, Flipped Classroom, Team-based learning and others. One of the suggested techniques that been invented is the use of assignment to engage students in their self-learning and progress and enhance their problem-solving capabilities beside motivating their team working skills.

Assignment's writing has been used heavily in different experiences like for improving critical thinking in political science course. The authors concluded that through well-designed writing assignments, instructors can encourage students to reconsider concepts, critically evaluate assumptions, and undertake substantive revisions of their writing [6]. Another study by Hill M., et al. proved that successfully implemented a case-based assignment activity in a first-year medical school course have been contributed to their Self-Directed Learning, self-reflection and metacognition and that students perceived the activity as a valuable learning experience [7]. Also, Sahoo S1, Mohammed CA used research protocol writing 
assignment activity for Fostering critical thinking and collaborative learning skills among medical students [8].

In Fact, Research skills are required in all branches of medicine, even if they will not pursue a research career, physicians need to be lifelong learners, able to evaluate evidence and understand the process of scientific enquiry [9]. They may also be required to carry out their own research, and the training we provide should lay the foundations for them to develop their own rigorous and informed approaches to research [10].

Traditional way of teaching research course is generally taught in a culminating Factual knowledge in the early years of Family Medicine residency program, alongside non-explorative experiments. Opportunity for novel constructive research is very minimal. an extensive US study by Momsen., et al. in 2010, argues that although science students are exposed to research opportunities, they are predominantly assessed on factual content at lower cognitive levels, meaning that graduates are less equipped with transferable skills than they perhaps should be [11].

In the AMEE guide 69 [9]; Courses like research has been always seemed (across our residents) very tough theoretical and away from any pragmatism, this is may be referred to being compulsory, could be also due to skill' deficit within the instructors of the course, how to link between the research fields and the clinical and community fields through real examples maybe, and at the end of the course, we are not sure of the actual gaining of the residents for the research knowledge and limitations.

So, there is a pressing need to change the way resident learn how to conduct research from traditional methods of theoretical to practical, from spoon feeding to active learning and from lectures based top assignment based.

\section{Methods}

A review of literature of the best evidence on use of assignmentbased learning for adults learning, a description of the assignments, its development and its contents and a description of the process of learning by residents and their active role in their learning and a summary for the case study.

This is a qualitative study describing the introduction of assignment-based learning to the research methods course as part of family medicine residents and the requirement of the SCFHS.
- A description of what the status before the introduction of the assignments,

- A description of the assignments

- A description of the role of the residents and the instructors

- And a description of the impact and improvement of the resident's performance during the research methods course.

Review of current situation before implementation of the assignments for research course educational activities; so, Description of the situation before the implementation of assignments and specifically including the residents' engagements in education and learning.

\section{Use of focus group discussions}

Two groups will be formed: each is consisted of:

- 8 residents

- Moderator

- Analyzer

- The moderator will ask the following questions in the data collection form attached; to illicit residents' inputs and reactions towards engagement in the educational process and;

- The analyzer will record inputs and reactions of residents

- Collection of data from residents and their feedback after use of assignments to improve learning in research course.

- The input and perception of residents will be analyzed qualitatively to describe impact and improvement after assignments application for improving residents engagement in educational and learning.

Data collection form used during two groups of resident's discussions:

\section{Session 1: Before implementation of Assignments}

The interviewer will explain the purpose of the group discussion and it's anonymous and it will not be used by any how except for the current research:

- Q1. What level you are in?

- Q2. Are you comfortable with current research activities? 
- Q3. Do you feel there is a need to modify how you learn?

- $\quad$ Q4. What are the priority areas for improvement?

- Q5. Do you agree on assignments as of learning improvement?

- Q6. Are you willing to collaborate to implement the assignments?

- Q7. How to improve residents' engagement in process of teaching research?

Session 2: After implementation of Assignments in research activities

The interviewer will explain purpose for the group discussion, and its anonymous, and remind the group of residents with their inputs and perceptions collected from session 1,

- Q1. What level you are in?

- Q2. Are you comfortable with current assuagements in research activities?

- Q3. Do you feel there is a need to modify how you learn?

- Q4. What are the priority areas for improvement?

- Q5. Did any notice impact of implement the assignments?

- Q6. What is the important improvement you notice to education and learning?

\section{Results}

Pre assignment

The conclusion of the residents was varying from carelessness about conducting research to feeling discouraged to work on research projects.

The consensus were the followings:

- That research is a vague issue

- Why to work on research while we have other pending educational issues

- We are not interested in research, we are interested to learn patient encounter and care issues

- As for the answers to questions:

- Q1. What level you are in?
All are R2 in year 2017

- Q2. Are you comfortable with current research activities?

6/8 students were not satisfied

- Q3. Do you feel there is a need to modify how you learn?

5/8 students agree for the need to change how research are done during residency training

- Q4. what are the priority areas for improvement?

$6 / 8$ residents mentioned that its needing a overhauling for the process of research training

- Q5. Do you agree on assignments as of learning improvement?

$7 / 8$ residents explained they had no exposure to assignments methods in research training

- Q6. Are you willing to collaborate to implement the assignments?

6/8 were careless to change

- Q7. How to improve residents' engagement in process of teaching research?

4/8 residents requested a separate time for the research activity.

Post establishment of assignment-based research training:

The conclusion of the residents was varying from raised interest in research and more care about conducting research to feeling value to work on research projects.

The consensus were the followings:

- That research is interesting and joy

- There is an overall culture of competition on research participation and research publications

- Its concurrent with the SCFHS requirements to complete a research proposal by End of R1, so assignments help to complete the requirement.

- Many explained the interest to complete the research work 
up to publication, not only the basic requirement which is the proposal and IRB approval

- As for the answers to questions:

- Q1. What level you are in?

All are R2 in year 2018

- Q2. Are you comfortable with current research activities?

8/8 students were satisfied

- Q3. Do you feel there is a need to modify how you learn?

7/8 students agree on that the change how research to assignment-based learning is much better than lecture based during residency training

- Q4. what are the priority areas for improvement?

5/8 residents mentioned that current the process of research training of being assignment-based is interesting and should be continued as basis for future training on research methods.

- Q5. Do you agree on assignments as of learning improvement?

6/8 residents explained they had interesting experience to assignments methods in research training

- Q6. Are you willing to collaborate to implement the assignments?

8/8 were interested to collaborate and continue same process of assignment-based training on research methods in different residency years.

- Q7. How to improve residents' engagement in process of teaching research?

$4 / 8$ residents requested to be more than one resident on one research topic. $3 / 8$ requested support from the training institution on support for scientific writing and publishing issues and finances.

\section{Discussion}

A physician must be a lifelong learner, understand the scientific method, and be able to evaluate evidence, regardless of whether he or she intends to pursue a research career [9]. Critical thinking has been enhanced extensively by assignment writing. Well-designed assignments can stimulate residents to reconsider concepts, critically evaluate assumptions, and revise their writing [6]. In this study, the aim was to see whether assignments during a residency course that includes training in research methods can enhance the learning process of family medicine residents and improve their satisfaction and understanding of research concepts and methods.

The satisfaction of learners improves performance, and many scholars use satisfaction as a critical component to evaluate their performance [12]. Our residents prior to initiation of assessment based research training were not aware about the implication and benefits of assignment-based style in improving their research understanding. However, after implantation all the residents were comfortable and satisfied with the new method of research teaching, and they encourage the continuation of it for future residents. Furthermore, their satisfaction with the experience increases their willingness to collaborate in their assignments. Almost all the residents agreed that the new methods were better than the old traditional method and helps to improve their performance. And they were eager to collaborate more in research assignments following the implementation of the new assignment and assessment style.

Finally, the responses of participants support the notion that assignment-based training facilitates the improvement of residents' perceptions of the need, fosters their interest, and enhances their performance during the research training. This shows the effectiveness of this new approach in improving the residents' learning experience.

\section{Conclusion}

Implementation of assignment-based training on research training for family medicine resident had a positive impact on residents' perception towards the need, practice, and interest in completing research training as a requirement for the SCFHS.

\section{Bibliography}

1. Kearsley, G. "Andragogy (M. Knowles). The theory into practice database" (2010).

2. Adult Learning by Assignment: London School of International Business. 
3. Knowles Malcolm. "The adult learner: a neglected species". (3rd ed.). Houston: Gulf Pub. Co., Book Division (1984).

4. Bohonos Jeremy. "Appreciating the Experiences and Expertise of Adult Students". Journal of College Orientation and Transition 20.2 (2013): 133.

5. https://www.iwb.net.au/wp-content/uploads/2017/12/ FlipConAusFlyer-v1.pdf

6. Gamze Çavdar. "Learning through Writing: Teaching Critical Thinking Skills in Writing Assignments". The Teacher 45.2 (2012): 298-306.

7. Hill M. "Implementation, and evaluation of a self-directed learning activity for first-year medical students". Medical Education Online 25.1 (2020): 1717780.

8. Sahoo S and Mohammed CA. "Fostering critical thinking and collaborative learning skills among medical students through a research protocol writing activity in the curriculum". Korean Journal of Medical Education 30.2 (2018): 109-118.

9. Anita Laidlaw. "Developing research skills in medical students: AMEE Guide No. 69". Medical Teacher 34.9 (2012): 754-771.

10. Helen R Watson. "Research skills in medical education". Med Ed Publish, Version 1 (2018).

11. Momsen J L., et al. "Just the Facts? Introductory Undergraduate Biology Courses Focus on Low-Level Cognitive Skills"'. CBELife Sciences Education 9.4 (2010): 435-440.

12. Ko WH and Chung FM. "Teaching Quality, Learning Satisfaction, and Academic Performance among Hospitality Students in Taiwan". World Journal of Education 4.5 (2014).

\section{Assets from publication with us}

- Prompt Acknowledgement after receiving the article

- Thorough Double blinded peer review

- Rapid Publication

- Issue of Publication Certificate

- High visibility of your Published work

Website: www.actascientific.com/

Submit Article: www.actascientific.com/submission.php

Email us: editor@actascientific.com

Contact us: +919182824667

Citation: Mostafa Kofi., et al. "Improving Research Training Using Assignments for Family Medicine Residents in Saudi Arabia". Acta Scientific Medical Sciences 6.1 (2021): 82-87. 